\title{
Openphone User Engagement And Requirements Solicitation in Low Literacy Users
}

\author{
T. Jama Ndwe ${ }^{1}$, Etienne Barnard ${ }^{2}$, Mqhele Dlodlo ${ }^{3}$, Daniel Mashao ${ }^{4}$ \\ Christiaan Kuun ${ }^{5}$, Aditi Sharma ${ }^{6}$ \\ ${ }^{1}$ CSIR, South Africa, JNdwe@ csir.co.za \\ ${ }^{2}$ CSIR, South Africa, EBarnard@csir.co.za \\ ${ }^{3}$ University of Cape Town, South Africa, Mqhele.Dlodlo@uct.ac.za \\ ${ }^{4}$ SITA, South Africa, Daniel.Mashao@sita.co.za \\ ${ }^{5}$ CSIR, South Africa, CKuun@csir.co.za \\ ${ }^{6}$ CSIR, South Africa, ASharma1@csir.co.za
}

\begin{abstract}
The OpenPhone project aims to design an Interactive Voice Response (IVR) health information system that enables people who are caregivers for HIV/AIDS infected children to access relevant information by using a telephone in their native language of Setswana in Botswana. The system lowers accessibility barriers since it is accessible to illiterate users and the community of the blind. The design utilizes usability engineering methodology in order to ascertain that the end product is usable, efficient, effective and satisfactory to the targeted users who are predominantly females, ranging from semi-literate to illiterate adults but nevertheless numerically literate. The paper describes the methodologies that were used to obtain information from the target user population. Based on the information gathered, we are now able to begin the initial design of the OpenPhone system.
\end{abstract}

Keywords: Usability engineering, User Requirements, Participatory Design, OpenPhone, Botswana-Baylor Children's Clinical Centre of Excellence

\section{Introduction}

In order to develop a system that meets the users' anticipation of the system the developers have to depend on the information that is provided by the users or anticipated users of the system (Lynch and Gregor, 2004). In this paper the development of collaboration between the researchers and the anticipated target users of the OpenPhone system is presented. Potential users were requested and encouraged to participate in the design process as a strategy to ensure that the product designed meets their needs and is usable to them. User engagement is particularly important for this targeted user group because of a cultural aspect, "where a questioner invariably gets positive answers as a matter of politeness even 
if these are not actually true! Criticism is seen as a sign of disrespect" (Blake and Tucker, 2006).

\section{Caregiver focus groups}

The focus group approach was chosen as the primary methodology for acquiring initial user needs from the intended target users of the OpenPhone system. This methodology is pragmatically appropriate for this particular user group because focus groups:

- Do not discriminate against people who cannot read or write

- Can encourage participation from those who are reluctant to be interviewed on their own (such as those intimidated by the formality and isolation of a one to one interview)

- Can encourage contributions from people who feel they have nothing to say (Kritzinger, 1995).

These characteristics about focus groups and the use thereof fit the intended user population well in the milieu of the unique situation and challenges of this user population as discussed in section 1 and in the abstract.

The caregiver focus group meetings had 3 primary objectives.

- The first was to study the user characteristics in order to compile and develop a user profile. The first stage in the usability process is to study the product's intended users (Nielsen, 1992).

- The second was to allow the targeted users to voice their opinions as to what concerns would they like the proposed system to address.

- The third objective was to engage the targeted users to form a coalition in the design of the system through participatory design which intends to involve the targeted users in all phases of the project. This particular focus group engagement was focused on introducing the system to the target user population and gathering opinions, beliefs, and attitudes about issues of interest about the proposed system.

There were 11 participants on the first day and 16 on the second and were all female with only one male participant on the second day. Both sessions started with a welcome speech that also thanked the participants for attending the focus groups. All conversations and interactions with the caregivers were conducted in Setswana with the aid of 2 moderators who are both fluent in English and the local language of Setswana. One of the moderators was a local Botswana citizen and resident which has helped the research team in comprehending the local cultural nuances which would not have been understood by any other means, not even by the other moderator who is fluent in Setswana but not a resident nor a citizen of Botswana. 
The moderators made it clear to the participants that the research needs to learn from them as to what was needed to be addressed by the proposed system. The moderators informed the participants that the system to be built would only succeed if the participants, who will be the users of the system, collaborated and partnered with the research team in building the system and that the team was respectfully asking for their cooperation. The research team is fully aware of the limitations of how much the participants can contribute to the design of the system, but nevertheless, the research team regards the participants, who are future end-users of the system, as experts developing and defining tools for their own use within their own environment (Schuler and Namioka, 1993).

The participants then enthusiastically engaged in the discussion and brainstorming of concerns that they felt the OpenPhone system should address. The discussion was again conducted by the 2 moderators and 2 observers were taking notes of the discussion. Both focus group sessions were recorded on a computer with a microphone connected to it. Their enthusiasm was evidenced by the fact that they would chat amongst each other and discuss amongst themselves what they think is necessary to be available on the proposed system before giving their views to the moderators. Naturally some participants were more talkative than others but the moderators encouraged those who were less talkative by engaging them in the discussion and asking them what they consider important and should be made available in the proposed system. The focus groups took an average of 105 minutes each and at the end of the focus groups the participants were then thanked for their participation.

\section{Summary of findings}

Unexpected issues on social services such as government grants were brought up by the participants but unfortunately these issues cannot be addressed directly by the system. Issues that were contemplated by the designers as of high importance such as caregiver psychological support were perceived as of low priority by the participants. When the participants were probed about this issue they stated that they get psychological support through strong immediate and extended family support. This support can also be communal which is typical of the Tswana culture whereby family and close community members are supportive towards other community members especially in the rural areas where those communal values are still maintained.

\section{Benefits of the focus group meetings}

Conducting the focus group meetings has had an impact on the designers' beliefs in terms of testing the designers' general assumptions whereby some of the information that the designers imagined as important to the caregivers was not viewed as such by the participants. This supports Robinson's notion of difficulty 
in anticipating a system's use in its actual applied work environment (Robinson, 1993). The meetings have enabled the designers to gain the targeted users' inputs on what their information requirements are and to eliminate unnecessary elements that the users don't need in the proposed system, which they have other ways to cope with. The meetings have also helped the design team in building a persona which is a model user that the design team creates to help understand the objectives, needs, and behaviours of the target users who will use the system interface. Benefits of creating an OpenPhone persona are:

- Creating a persona has assisted the designers to approach the design more objectively, with their target user in mind, instead of their own views and beliefs. Instead of asking, "How would I use this system?" the designers are now asking, "How would the caregivers use the system?"

- In using the persona as the target character, the designers are more capable of identifying how the caregivers will interact with the design. This enables the designers to gain an insight about the design and system usage that they wouldn't have gained in any other way.

- Puts all the design team members on the same page as far as to who the design is intended.

- It enables designers to put themselves in the shoes of the target users.

In sum the persona helps the researching designers make a smooth transition between user requirements and the design, which will benefit the overall design of the system. The created persona is a primary persona and is expected to evolve as the designers gain more knowledge about the targeted users of the technology.

\section{Conclusions}

User needs gathering is a way of animating and furnishing influential information into the design process that will have impact in the manner in which the system is designed. In conducting real user observations the researchers' findings get to be based on realities, not preconceptions. Users bring about things that the researchers would erroneously consider unimportant in a focus group. On the other hand users also remark on the things that the researchers thought to be essential but users don't see to be beneficial to them as they have other ways of dealing with such issues beyond the capabilities of the system.

The contacts made with the actual prospective users enable strong relations between the users and the researchers to be formed. These relationships are envisaged to create trust and understanding between the parties in order to devise a bond with common goals of designing a truly usable system.

In a new and modern design the designers may be mislead into assuming that there is no need for user studies because the product idea is new and groundbreaking to the target users and therefore there is no useful information that can be 
provided by the users. On the contrary, it is essential to observe and interview people in order to understand how they cope in doing things the traditional way before bringing in the new way of doing things. Through the interactions with target users the researchers may discover that they are solving the wrong problem, or that they have overlooked some other more important problems that need to be solved. Researchers may also find that there are features of the old way of doing things that work well which need to be reserved and incorporated in the new design. For example, in the OpenPhone system there is a need to use the same language and terminology that is usually used by the lecturing staff during the lectures at the Botswana-Baylor Children's Clinical Centre of Excellence as the users are accustomed to those terms and language and not the scientific terms and language as acquired by researchers from formal literature. The Botswana-Baylor Children's Clinical Centre of Excellence is the clinic where the caregivers normally go to get information on giving care to HIV infected children, where the focus group meetings were held.

We have found out that although the participants lack knowledge about technical matters on how to build an appropriate IVR system, they are rich in common sense knowledge about their needs and their typical concerns on caregiving issues. Both scientific and common sense knowledge is important in formulating a holistic solution.

Based on these focus group meetings and the persona that has been created the designers are able to turn the requirements information into functional specifications before beginning the initial design of the system.

\section{References}

Blake, H. and Tucker, D. 2006. User interfaces for communication bridges across digital divide. AI Soc. 20, 2 (Feb. 2006), 232-242.

Kritzinger, J. (1995) Qualitative Research: introducing focus groups. BMJ 311:299-302.

Lynch, T. and Gregor, S. 2004. User participation in decision support systems development: influencing system outcomes. Eur. J. Inf. Syst. 13, 4 (Dec. 2004), 286-301.

Nielsen, J., "The usability engineering life cycle," Computer, vol.25, no.3, pp.1222, Mar 1992.

Robinson, M. 1993. Design for unanticipated use.. In Proceedings of the Third Conference on European Conference on Computer-Supported Cooperative Work (Milan, Italy, September 13 - 17, 1993). G. de Michelis, C. Simone, and K. Schmidt, Eds. ECSCW. Kluwer Academic Publishers, Norwell, MA, 187202.

Schuler, D. and Namioka, A. (1993). Participatory design: Principles and practices. Hillsdale, NJ: Erlbaum. 